\title{
Retromolar Intubation - A Non-Invasive Approach of Airway Management during Maxillofacial Surgery.
}

\author{
Kumkum Gupta ${ }^{1}$, Bhanupriya Agrawal ${ }^{2}$, Prashant K Gupta ${ }^{3}$, Salony Agarwal ${ }^{4}$, Akshat Trivedi ${ }^{2}$, Kopal Gupta ${ }^{2}$ \\ ${ }^{1}$ Professor, Department of Anaesthesiology and Critical care, ${ }^{2}$ Resident, Department of Anaesthesiology and Critical care, ${ }^{3}$ Director Professor, Department \\ of Radio diagnosis and Imaging, ${ }^{4}$ Associate Professor. Department of Anaesthesiology and Critical care, Subharti Medical College, Swami Vivekanand \\ Subharti University, NH-58, By Pass Road, Meerut-UP, India.
}

\section{Abstract}

Background: Patients of maxillofacial injury require precise attention for securing and protecting the shared airway. Moreover, intraoperative assessment of dental occlusion and maxillomandibular fixation is often required for meticulous reduction of facial fractures, which makes the presence of oral endotracheal tube unfeasible. The aim of the study was to evaluate the retromolar placement of tracheal tube to secure the airway in patients of maxillofacial injury. Subjects and Methods: Thirty seven adult consenting patients of ASA physical status I and II of either gender aged 20 to 58 years who met the inclusion criteria, were enrolled for this prospective cohort study. After induction, orotracheal intubation was done with flexometallic tube, then the tube was grasped with gloved fingers and mobilize to retromolar apace, fixed with elastic tap at the angle of mouth and oropharyngeal packing was done. After completion of surgery, patients were extubated after proper suction of oral cavity and removal of oropharyngeal pack. There was no intraoperative or postoperative complication due to anesthetic technique. Results: Retromolar intubation was performed successfully without any difficulty in all patients. Intraoperative assessment of dental occlusion and maxillomandibular fixation could be performed without any noticeable changes in airway pressure or visible tube kinking. No anesthetic or surgical complications were encountered in any patient. Conclusion: Retromolar intubation efficiently secured the airway and provided uninterrupted operating field to the surgeon during maxillofacial surgery. Lack of any adverse events encouraged us to present the advantages of retromolar intubation on the basis of our own experience.

Keywords: Maxillofacial Injury; Flexometallic Tracheal Tube; Maxillomandibular fixation; Retromolar Intubation.

Corresponding Author: Prof (Dr) Kumkum Gupta, 108-109, Chanakyapuri, Shastri Nagar, Meerut-250004 U.P. India.

Received: January 2019

Accepted: February 2019

\section{Introduction}

Patients with complex maxillofacial injuries require an effective and secured technique for airway management with an uninterrupted surgical field. ${ }^{[1]}$ Moreover, intraoperative assessment of dental occlusion and maxillomandibular fixation is often required for precise reduction of facial fractures, which poses great challenge to anaesthesiologist. Though, tracheal intubation with cuffed endotracheal tube is mandatory to secure and protect the airway from blood and debris during maxillofacial surgery, but in such patients airway management using oral endotracheal tube is potentially rule out while nasal route is contraindicated due to nasal bone/ basal skull fracture, intranasal pathology or bleeding diathesis. ${ }^{[2]}$

The tracheostomy could provide a secure airway with no surgical interference but was not considered because of it invasiveness and association with subglottic stenosis, injury to lingual nerve/esophagus, speech and swallowing difficulties. The other available intubation technique was submental intubation, which avoids the need for short term tracheostomy but is associated with oro-cutaneous fistula, injury to sublingual/ submandibular gland, hypertrophic scarring and infection. ${ }^{[3,4]}$
Thus current strategies for airway management in patients of maxillofacial injuries were neither effective nor reliable for intraoperative restoration of occlusion of jaw after maxillo mandibular reduction. Therefore an alternative approach was desirable. Many studies have documented that endotracheal intubation through the retromolar space is effective and reliable technique for restoration of intraoperative dental occlusion and it is neither invasive nor interferes during intraoral surgery. ${ }^{[5]}$

This prospective cohort study was designed to evaluate the clinical efficacy of using the retromolar space for endotracheal tube placement by conventional method to achieve intraoperative restoration of dental occlusion and maxillomandibular fixation for meticulous reduction of facial fractures, as primary objective. Any complication of technique or during surgery would be secondary objective of the study.

\section{Subjects and Methods}

After approval of the protocol by the Institutional Ethical Committee, written informed consent was obtained from all patients. The study was conducted on thirty seven adult 
patients of American Society of Anaesthesiologist (ASA) physical status I and II of either gender, aged 24-56 years, weighing 50 to $75 \mathrm{~kg}$, and scheduled for elective maxillofacial surgery under general anesthesia.

Patients with history of any cardiac or respiratory disease, hypertension, obesity (BMI $>26 \mathrm{~kg} / \mathrm{m} 2$ ), hepatic or renal dysfunction, endocrinal or metabolic disorders were excluded from the study. Disrupted laryngotracheal anatomy and restricted retromolar space were other exclusion criteria.

All the patients underwent preanesthetic evaluation, including history, general physical examination, systemic examination and their biochemical investigations were also reviewed. Preoperative airway evaluation was focussed on jaw opening, mask fit, neck mobility, macroglossia, dental pathology, nasal patency and the existence of any intraoral lesions or debris.

\section{Anesthetic Technique:}

All selected patients were given tablet Alprazolam $0.25 \mathrm{mg}$ and tablet Ranitidine $150 \mathrm{mg}$ orally the night before surgery and were kept fasting for 6 hour prior to surgery.

On the day of surgery, all patients were given glycopyrrolate $0.2 \mathrm{mg}$ intramuscularly, $30 \mathrm{~min}$ prior to induction of anesthesia. On arrival to operation room, all patients were placed supine with the support of pillow. Multipara monitor was attached to monitor the baseline vital parameters of heart rate, systemic blood pressure, peripheral oxygen saturation $(\mathrm{SpO} 2)$ and electrocardiogram (ECG). An intravenous line was secured and lactate Ringer solution was stared at rate of 4-6 $\mathrm{ml} / \mathrm{kg} / \mathrm{h}$ and ondansetron $(4 \mathrm{mg})$, midazolam $(2 \mathrm{mg})$ and fentanyl $(2 \mu \mathrm{g} . \mathrm{kg}-1)$ was administered intravenously for premedication.

After pre oxygenation for 3 min with $100 \%$ oxygen via face mask, anaesthesia was induced with propofol $(1 \%) 2 \mathrm{mg} / \mathrm{kg}$, till loss of verbal command. Following adequate bag and mask ventilation with $100 \%$ oxygen, rocuronium bromide $0.08 \mathrm{mg} / \mathrm{kg}$ was administered and patients were manually ventilated with Sevoflurane and $100 \%$ oxygen. When double burst suppression was $95 \%$ or when Train of four (TOF) was zero, the patient's head was extended and neck was flexed to align the oral, pharyngeal and laryngeal axes. The direct laryngoscopy was performed using Macintosh curve blade laryngoscope and intubation was performed with proper sized well lubricated flexometallic nylon reinforced cuffed endotracheal tube. The orotracheal tube was then grasped with gloved fingers and mobilize to retromolar apace and fixed with elastic tap at the angle of mouth, after ensuring bilateral equal air entry. The oropharyngeal packing was accomplished to minimize the amount of blood or debris reaching the larynx and trachea.

Anesthesia was maintained with Sevoflurane, nitrous oxide $60 \%$ in oxygen by mechanically ventilating the patient using close circuit with a tidal volume of $10 \mathrm{ml} / \mathrm{kg}$, respiratory rate of 12 breaths/min and I: E ratio of 1:2 in volume $\square$ controlled mode. The tidal volume and ventilatory frequency were adjusted to maintain EtCO2 between $35-40 \mathrm{~mm} \mathrm{Hg}$. The degree of muscle relaxation was maintained with supplemental dosages of rocuronium bromide $(0.02 \mathrm{mg} / \mathrm{kg})$. Patients were assessed for changes in the heart rate and blood pressure before induction, after intubation and then after at every 5 min interval till end of surgery and post extubation.
Intraoperatively, systolic blood pressure was maintained within $20 \%$ of preoperative value. Any bradycardia or tachycardia, hypotension or hypertension was managed as required, by adjusting the dial concentration of Sevoflurane or infusion rate of lactate Ringer solution.

At the end of surgery, the retromolar tracheal tube was converted back to orotracheal tube and oropharyngeal pack was removed after adequate suction of oral cavity. The residual neuromuscular blockade was antagonized with appropriate doses of neostigmine $(0.05 \mathrm{mg} / \mathrm{kg})$ and glycopyrrolate $(0.01 \mathrm{mg} / \mathrm{kg})$. After assessment the levels of consciousness and adequacy of reflexes, patients were extubated when respiration was adequate and were able to obey simple commands.

Patients were transferred to post-anesthesia care unit and monitored for any oral bleeding, any airway problems or any technique related adverse effects.

\section{$\underline{\text { Sample size }}$}

Preliminary sample size was decided in consultation with statistician and was based on previous studies, which indicated that approximately 32 patients should be included in order to ensure power of $80 \%$ and alpha error of 0.05 with confidence limit of $95 \%$ for better validation of results. Assuming a 5\% drop out rate, a total of 37 patients were incorporated in the study.

\section{Results}

Patient's demographic data included 37 patients (26 males and 11 females) with a mean age of $27.4 \pm 7.53$ years $(24$ to 56 years) and mean weight of $63.45 \pm 8.71 \mathrm{~kg}$ (50 to $75 \mathrm{~kg}$ ). The standardised technique of general anesthesia and orotracheal intubation was used and retromolar intubation with conventional method was performed in every patient without any difficulty with a flexometallic cuffed endotracheal tube. The average time taken to position the tube into retromolar space was less than a minute. During procedure, no difficulty was encountered in positioning the tracheal tube in either retromolar space.

Intraoperatively, the retromolar intubation allowed adequate dental occlusion thus rendering maxillomandibular fixation feasible after precise reduction of facial fractures. The airway was never compromised and no episode of arterial desaturation occurred during procedure and perioperative period. All patients were extubated by standard method.

In two patients, there was accidental disconnection of tube from the breathing circuit intraoperatively, which was immediately recognized and reconnected to breathing circuit.

\section{Discussion}

Maxillofacial trauma needs particular vigilance of anaesthesiologists due to 'shared airway surgery'. Moreover, surgery often requires manipulation and movement of the head, neck and mouth, thus creating the risk of obstructing, transecting, and disconnecting or dislodging the tube. An oropharyngeal pack is often indicated due to risk of soiling by blood and debris.

The choice of airway in maxillofacial surgery is determined 
by patient factors, surgical requirements and anaesthetist preferences. But till date no consensus could be established as to which is the best technique to secure the airway. A cuffed endotracheal tube provides the highest level of airway protection in shared airway surgery. ${ }^{[1]}$ In patients of maxillofacial trauma, the oral endotracheal is potentially rule out as it does not allow intraoperative assessment of dental occlusion. Though nasal intubation technique is the mainstay of shared airway surgery but this route cannot be opted due to nasal bone fracture or intranasal pathology.

Tracheostomy could provide a secure airway with no surgical interference but it may not be considered due to its invasiveness and its association with its few inherited problems. Another available intubation technique is submental intubation, which avoids the need for short term tracheostomy but it is associated with its own drawbacks. Thus, retromolar intubation can be a better option to secure airway in such patients, as it avoids the need of any surgical airway when intraoperative dental occlusion is to be assessed. Retromolar intubation is non-invasive, less time consuming procedure and avoids the problems of other available modalities. ${ }^{[6]}$

Retromolar intubation was first described as an alternative route for oral or nasal intubation by Martinez et al. ${ }^{[7]}$ Since the first application of this technique, many researchers have studied the clinical use of this procedure. Halsnad et al used the retromolar space for intubation in patients of mandibular fracture while Gibbons et al placed the tracheal tube in retromolar space in patient of complex craniofacial trauma. ${ }^{[8,9]}$ They all reported very few rates of complications. The retromolar space is located between the distal aspect of the last molar and the anterior edge of the ascending ramus of the mandible where it crosses the alveolar margin. It is bounded anteriorly by last erupted molar tooth, posteriorly by the ramus of mandible, superiorly by the maxillary tuberosity and inferiorly by the retromolar trigone area.

The appropriateness of this space can be judged by placing a gloved index finger behind distal molar and asking the patient to close the mouth slowly. No compression on the finger indicates enough retromolar space. The dental pantomogram may be helpful for evaluating the adequacy of the retromolar space. If retromolar space is not adequate, dental occlusion may not be possible and intraoperative fixation of jaw fractures cannot be achieved. ${ }^{[10]}$

Dental pan-tomograms have reported a mean height of 17.9 $\mathrm{mm}$ for the right retromolar space and $18.1 \mathrm{~mm}$ for left retromolar space with reported mean width of $17.5 \mathrm{~mm}$ for right space and $16.5 \mathrm{~mm}$ for left space, which suggested that the retromolar space is adequate enough to readily accept an $8 \mathrm{~mm}$ endotracheal tube with an outer diameter of $10.8 \mathrm{~mm}$. Because the existence of two retromolar spaces, in the presence of loose tooth or retromolar trigone lesion on one side, the uninvolved contralateral side can be safely used for tube placement.

During maxillofacial surgery, the tracheal tube may be subject to direct or indirect pressure so it is appropriate to use flexometallic reinforced tube to prevent kinging or occlusion of the tube during head or neck movements. Unlike the technique described by Martinez et al, the need of a flexometallic tube and fixation by wire ligature in a figure of eight pattern is not always necessary. ${ }^{[7]}$ In the present study, the orotracheal tube was fixed with elastic tap at the angle of mouth, and intraoperatively, dental occlusion could be achieved in all patients without any airway related complications.

There are two technique for retromolar intubation; conventional technique and Bonfils retromolar fiberscope technique. Bonfils retromolar fiberscope is a semi-rigid optical stylet with an angled end and it was not available in our institution. ${ }^{[11-13]}$

In the present study, we did the retromolar intubation by conventional technique which allowed adequate dental occlusion and rendering the fixation of jaw fractures. Restoration of adequate dental occlusion is the important step prior to surgical fixation of fracture segments by plates and screws. Dutta et al observed that there is great variation in the retromolar space in patients where third molar is impacted or fully erupted. ${ }^{[14]}$ Martinez et al described that if third molar is present whether erupted or unerupted, it may be extracted before performing a semi-lunar osteotomy large enough for the tracheal tube to lie below the occlusal plane. During osteotomy, the internal mucoperiosteal plane is protected to prevent injury to lingual nerve. This technique involves destruction of bony anatomy which can be used for fractures or for fixation devices. Martinez et al further described that if the retromolar space is not adequate then after orotracheal intubation with flexometallic tube, an angled retromolar incision can be made in the mandibular trigone area.

Another disadvantage of retromolar intubation is that tracheal tube can interfere with the main surgical field that might be partially draped. The tracheal tube can also interfere with positioning and application of dental fixation devices, especially in patient with pan facial trauma. Sometimes, fixation of flexometallic tube by wire ligature can deform the tracheal tube.

Retromolar intubation is feasible alternative to the invasive methods, moreover it is economical, less time consuming and associated with fewer complications. Sometimes space consumed by oral endotracheal tube can interfere with the application of dental fixation devices, and surgical field can be compromised, especially in cases of bilateral maxillary / mandibular fractures. The efficacy of retromolar intubation in patients with pre-existing tempromandibular dysfunction and its use for long term postoperative use is doubtful.

Despite these limitations, retromolar intubation can be considered an excellent alternative technique when temporary dental occlusion by inter mandibular or inter maxillary fixation is required as it rule out the need for any invasive airway management and complications related to them.

\section{Conclusion}

Airway management of maxillofacial injuries is one of the most challenging issue to anaesthesiologists. Retromolar intubation offers several advantages over the other surgical procedures like sub-mental intubation and tracheotomy, especially in craniofacial, orthognathic, oncologic and trauma surgeries involving intraoperative dental occlusion. 


\section{References}

1. Raja M, Iyer R, Thaller S. Airway Management in Patients with Facial Trauma. Journal of Craniofacial Surgery 2009; 20: 21-3.

2. Lee SS, Huang SH, Wu SH, Sun IF, Chu KS, Lai CS, et al. A review of intraoperative airway management for midface facial bone fracture patients. Ann plast Surg 2009; 63:162-6.

3. Caron G, Paquin R, Lessard MR, Trepanier Ca Landry PE. Submental endotracheal intubation: An alternative to tracheostomy in patients with midfacial and panfacial fractures. J Trauma 2000; 48: 235-40.

4. Malhotra N, Bhardwaj N, Chari P. Submental endotracheal intubation: A useful alternative to tracheostomy. Indian J Anaesth 2002; 46: 40002 .

5. Werther JR. Retromolar intubation. J Oral Maxillofac Surg 1998; 56: 305-06.

6. Hagberg CA. Special devices and techniques. Anesthesiol Clin North America 2002; 20: 907-32.

7. Martinez LJL, Eslava JM, Cebrecos AI,Marcos O. Retromolar intubation. J Oral Maxillofac Surg 1998; 56: 302-05.

8. Halsnad SM, Wilkins IA, Adlam DM. Oral endotracheal intubation in the management of mid fa cial fractures (letter). Br J Oral Maxillofac Surg 2005;43:190, 191.

9. Gibbons AJ, Hope DA, Silvester KC. Oral endotracheal intubation in the management of midfacial fractures. Br J Oral Maxillofac Surg 2003:41:259, 260 .

10. Arora S, Rattan V, Bhardwaj N. An Evaluation of the Retromolar Space for Oral Tracheal Tube Placement for Maxillofacial Surgery in Children. Anesthesia and analgesia 2006; 103: 1122-5.

11. Rungta N. Technique of Retromolar and Submental Intubationin in Facio - Maxillary Trauma Patients. Ind. J. Trauma. Anesthesia Critical care 2007; 8: 573-5.

12. Halligan M, Charters P. A clinical evaluation of the Bonfils intubation fibrescope. Anaesthesia 2003; 58: 1087-91.

13. Buehner U, Oram J, Elliot S, Mallick A, Bodenham A. Bonfils semirigid endoscope for guidance during percutaneous tracheostomy. Anaesthesia 2006; 61: 665-70.

14. Dutta A, Kumar V, Saha SS, Sood J, Khazanchi RK. Retromolar tracheal tube positioning for patients undergoing faciomaxillary surgery. Canadian journal of anesthesia 2005; 52: 341.

Copyright: () the author(s), publisher. Academia Anesthesiologica International is an Official Publication of "Society for Health Care \& Research Development". It is an open-access article distributed under the terms of the Creative Commons Attribution Non-Commercial License, which permits unrestricted non-commercial use, distribution, and reproduction in any medium, provided the original work is properly cited.

How to cite this article: Gupta K, Agrawal B, Gupta PK, Agarwal S, Trivedi A, Gupta K. Retromolar Intubation-A Non-Invasive Approach of Airway Management during Maxillofacial Surgery. Acad. Anesthesiol. Int. 2018;3(2):31-34.

DOI: dx.doi.org/10.21276/aan.2018.3.2.7

Source of Support: Nil, Conflict of Interest: None declared. 